\title{
De Lévi-Strauss à Haudricourt
}

Promenade architecturale en Savoie

From Lévi-Strauss to Haudricourt. Architectural walking in Savoy

\section{Emmanuel Désveaux}

\section{(2) OpenEdition}

Journals

Édition électronique

URL : https://journals.openedition.org/tc/5940

DOI : $10.4000 /$ tc. 5940

ISBN : 1952-420X

ISSN : 1952-420X

Éditeur

Éditions de l'EHESS

\section{Édition imprimée}

Date de publication : 5 septembre 2011

Pagination : 202-223

ISBN : 978-2-7351-1410-8

ISSN : 0248-6016

\section{Référence électronique}

Emmanuel Désveaux, « De Lévi-Strauss à Haudricourt », Techniques \& Culture [En ligne], 56 | 2011, mis en ligne le 30 novembre 2011, consulté le 29 septembre 2022. URL : http://journals.openedition.org/ tc/5940; DOI : https://doi.org/10.4000/tc.5940 


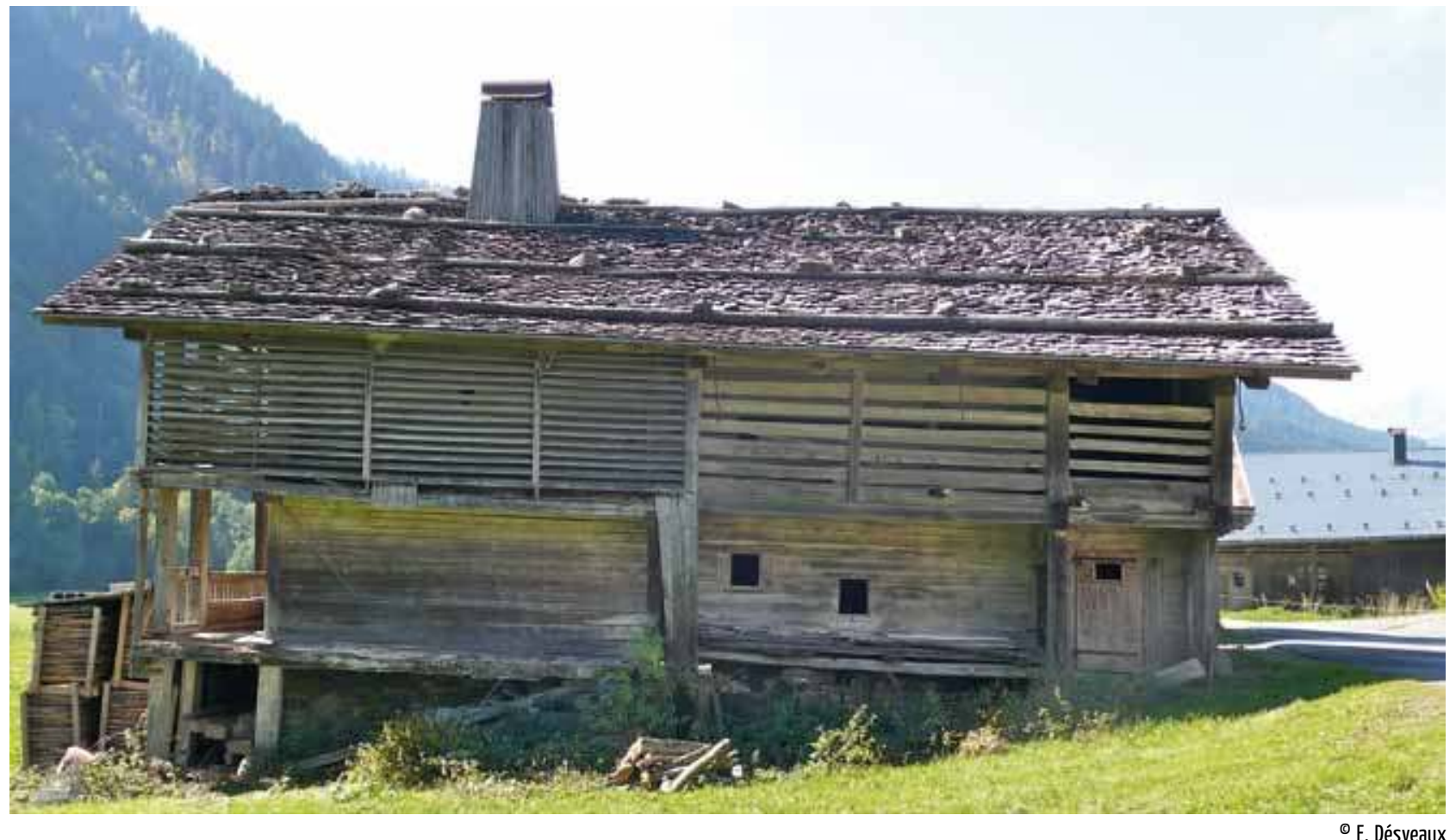




\section{DE LÉVI-STRAUSS À HAUDRICOURT}

\section{Promenade architecturale en Savoie}

Il existe, à cheval entre les départements de la Savoie et la Haute-Savoie, une aire géographique où prévaut un type de chalet ${ }^{1}$ bien caractérisé. Il s'agit en effet de ces constructions que les technologues appellent en madriers pièce sur pièce (Raulin 1977a : 35) ou encore, selon diverses expressions locales, à madriers, à encostes ou en bois carrés. On pourrait dire plus simplement: bois sur bois, car ce sont des bâtiments qui sont élevés selon une technique qui consiste à empiler des troncs entiers, plus ou moins équarris, les uns sur les autres. Ceux-ci, en vertu d'un système d'encoches ad hoc à leurs extrémités, s'emboîtent et se solidarisent (Figure 1). Le procédé se distribue à travers tout l'arc alpin. Il se rencontre ainsi de façon assez récurrente dans les hautes vallées suisses et autrichiennes et ce, jusqu'en Styrie (Weiss 1959 ; Tomasi 1977). Mais l'aire de distribution de la technique est beaucoup plus large puisqu'elle embrasse le domaine slave, jusqu'à ses propres confins, à savoir jusqu'au Caucase, et comprend également les régions scandinaves. Remarquons que, mis à part la zone de la Savoie septentrionale qui nous intéresse et sur les limites de laquelle nous reviendrons un peu plus loin en détail, la technique pièce sur pièce reste peu présente dans les Alpes françaises. On ne la retrouve que dans le Queyras, à St-Véran (Raulin 1977b : 50), et à Vallorcine, au-dessus de Chamonix, soit à proximité immédiate de la frontière suisse (Raulin 1977a : 105). Et encore, dans ce dernier village, le recours à cette technique se limite-t-elle aux bâtiments annexes, les greniers, appelés localement raccards. En tout état de cause, nous sommes alors déjà très proches de formes architecturales qui caractérisent le Valais immédiatement voisin.
"Assemblages: entiers et non-entiers" Héraclite, Fragments. (traduction de J. Bollack et H. Wismann). 
La technique en question a longtemps été interprétée en termes d'archaïsme ou de survivance. Aux yeux des chercheurs allemands notamment, elle apparaît comme une manière primitive de bâtir en bois qui aurait subsisté à la périphérie de l'aire germanique, dans les montagnes ou sur les marches de l'Est et du Nord. En effet cette vision évolutionniste se greffe chez eux à celle qui, dès la deuxième moitié du xix siècle, opère un partage entre architecture en bois, expression d'un génie bâtisseur propre au monde germanique, et architecture de pierre, synonyme de latinité. Cette thèse de l'essentialisme ethnique fait l'objet d'une critique radicale de la part de Richard Weiss dans les années 1950. Dans son magistral Häuser und Landschaften der Schweiz — dont l'argument se limite malheureusement à la seule Suisse — il assigne le choix du bois ou de la pierre comme matériaux de construction à des considérations strictement écologiques. Selon lui, grosso modo, plus on va vers le sud, moins on trouve de bois de qualité et plus la pierre s'impose, et inversement. On observe ainsi un passage progressif de la dominance d'un matériau à l'autre. Cela étant, Weiss évite d'aborder de front la question de la construction pièce sur pièce. Ce qui se comprend: son existence, ou son maintien, peut difficilement s'expliquer en raison de facteurs environnementaux — elle consomme à peine plus de bois que les techniques concurrentes dites en élévation sur poutres, ainsi que nous le verrons plus loin — tandis qu'un rejet de l'essentialisme ethnique entraîne nécessairement celui de ses éventuels antécédents.

Du côté français, la géographie humaine à la Vidal de la Blache se préoccupe peu, il faut le reconnaître, d'architecture vernaculaire. Elle n'y voit qu'une des facettes de son propre essentialisme, de nature géologico-historique ${ }^{2}$. Il faut attendre le renouveau des études sur les sociétés paysannes, dû en grande partie à l'impulsion que leur donna Lévi-Strauss à partir des années 1960 au Laboratoire d'anthropologie sociale grâce à Isac Chiva, pour qu'un regard scientifique se porte sur les manières paysannes de bâtir. On pense ici évidemment au vaste chantier lancé par Jean Cuisenier et Henri Raulin, censé couvrir à terme toute la France - il n'a jamais été achevé — et dont, par une sorte d'heureux hasard, la première région explorée s'avère avoir été la Savoie. L'optique se voulait essentiellement descriptive; d'un point de vue méthodologique, il s'agissait en outre de définir des protocoles descriptifs dont la validité transcenderait une typologie en termes de fonction, ou de « style » régional ${ }^{3}$. L'entreprise renvoie dos à dos essentialisme ethnique et adaptation écologique et traduit une sensibilisation au fait que ce type d'architecture constituerait en soi un langage formel ${ }^{4}$. Le structuralisme ambiant exigeait une mise à distance de toutes interrogations quant à l'antériorité de certaines formes par rapport à d'autres. Du coup, bien que toujours pensée en termes d'archaïsme, l'architecture pièce sur pièce de la Savoie septentrionale devenait, dans sa singularité, embarrassante. De fait, si Raulin (1977a) signale bien sûr son existence dans son ouvrage, il ne s'appesantit guère dessus, comme si le phénomène était en lui-même insignifiant.

Nous pensons le contraire. Nous proposons dès lors pour le traiter de faire appel à une démarche qui s'inspire directement de Lévi-Strauss. Mais il s'agira en l'occurrence du deuxième Lévi-Strauss - ainsi que nous l'avons défini dans nos travaux antérieurs (Désveaux 2001) —à savoir celui qui postule qu’une homogénéité de formes déployées dans un espace donné, à l'exemple des mythes des deux Amériques, constitue un corpus. Ce corpus est susceptible de donner lieu non seulement à des variations, mais aussi des transformations logiques. Celles-ci s'entendent comme des systèmes d'inversion dont la polarisation se traduit dans l'espace et se voit confortée par des états intermédiaires, eux aussi répétables dans l'espace. Surtout, ces transformations sont porteuses de sens, 
à la différence de la simple variation qui ne renvoie qu'à un principe de distinction. L'enseignement du deuxième Lévi-Strauss réside en outre dans une phénoménologie qui réserve sa meilleure place aux réalités de la nature comme matrice de la faculté intellective. D'un côté, cela nous permet de renouer avec l'intuition de Cuisenier et de Raulin selon laquelle l'architecture vernaculaire était un langage. D'un autre côté, cela nous incite à l'approfondir, car si la définition d'un langage est d'avoir une vocation sémantique, cette dimension restait étrangement absente de leur argument.

Dans un premier temps, nous tenterons de montrer en quoi l'architecture pièce sur pièce de la Savoie septentrionale fait corpus. Il nous faut écarter ici la tentation déterministe qui conduirait à renvoyer les variations observées à des adaptations optimales aux conditions écologiques ou à des modes d'organisation sociale dont les éventuelles différenciations seraient elles-mêmes observables d'un point de vue empirique. Dans un deuxième temps, nous mettrons en relief un système d'inversions au sein de ce corpus avec l'espoir d'être à même d'en dégager, dans une troisième partie, les significations. Somme toute, notre démarche n'est donc pas complètement inédite, puisqu'elle a au moins un précédent, en l'occurrence le travail de Richard Bucaille sur les pigeonniers de Limagne (Bucaille \& al. 1987).

Deux mots pour finir cette section sur nos motivations et nos méthodes d'enquête. La plupart des bâtiments qui nous intéressent est désormais modifiée, soit en vertu de la modernisation des exploitations agricoles, soit par mutation en résidences secondaires, voire principales mais alors en conformité avec une norme de l'habitat du Français « moyen » qui, dans tous les cas, dénature sensiblement les espaces bâtis. En bref, l'intégrité de l'architecture traditionnelle savoyarde est aujourd'hui gravement menacée, mais il subsiste encore des informateurs l'ayant connue dans son état « fonctionnel » (même si celui-ci n'a jamais été totalement figé). Or, ces derniers sont âgés. Il y a donc urgence à relancer l'enquête. Notre méthode, outre les entretiens, repose pour l'essentiel sur un arpentage le plus systématique possible du terrain. Nous avons ainsi approché plusieurs centaines de chalets et visité un bon nombre d'entre eux (nous disposons d'une base de données photographiques d'environ 700 entrées) ${ }^{5}$.

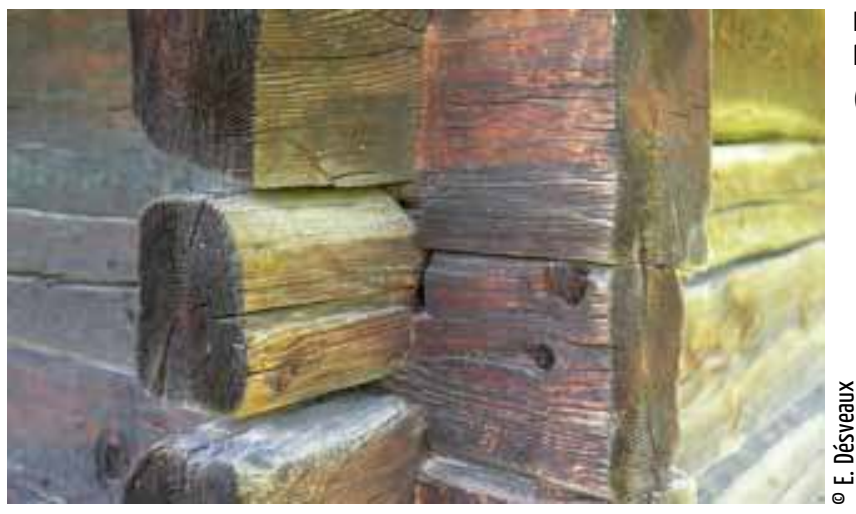
l'architecture pièce sur pièce.

(Fig. 1) 


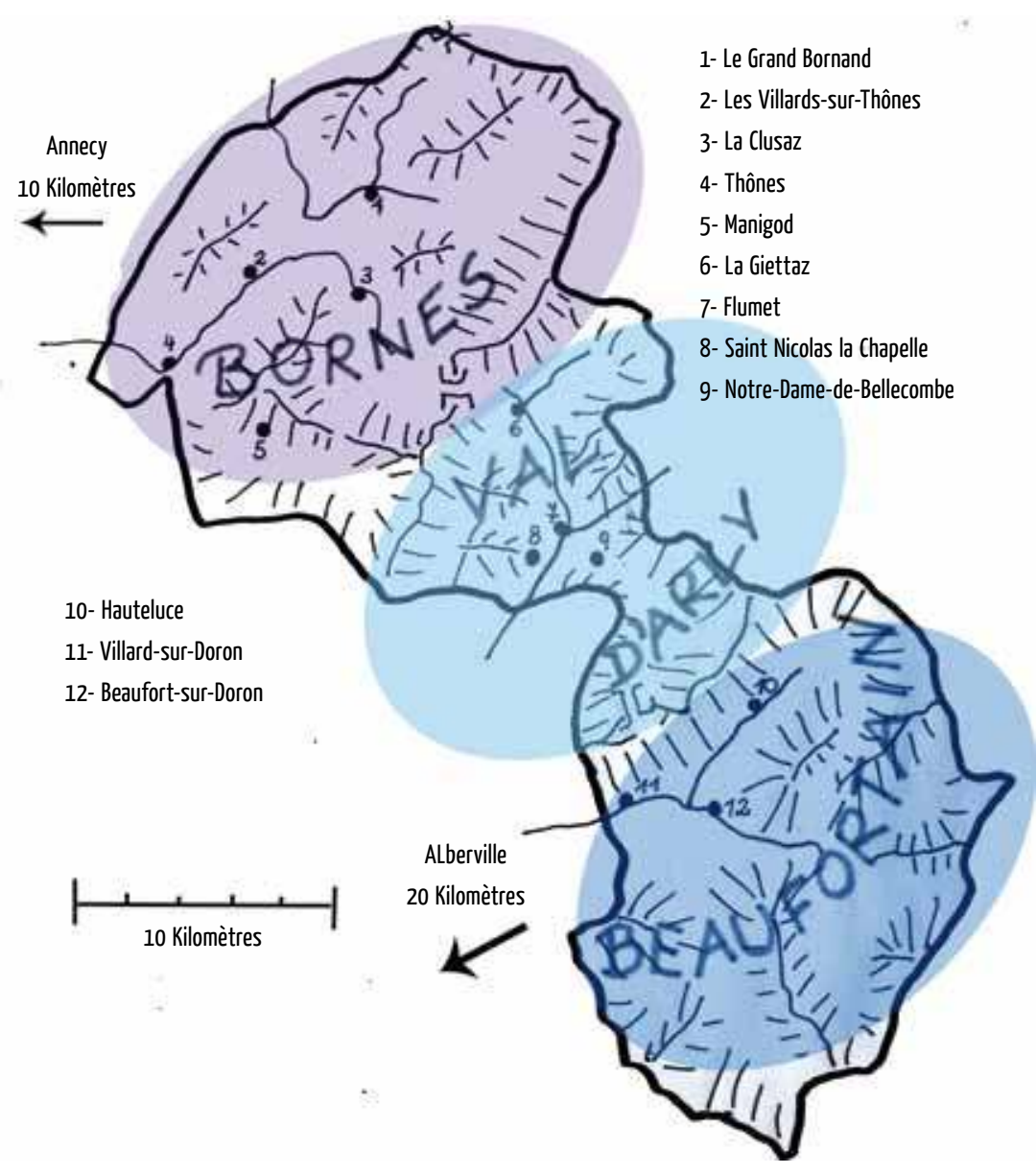

Aires de distribution de l'architecture en bois pièce sur pièce de Savoie. Relevé d'E. Désveaux.

(Fig. 2)

\section{Considérations géographiques}

Laire de distribution des chalets construits pièce sur pièce constitue un véritable nucleus qui couvre, du nord au sud, les deux tiers du massif des Bornes, la vallée médiane de l'Arly et enfin la totalité de la vallée du Doron et de ses affluents, à l'exception de sa partie inférieure ${ }^{6}$. Sur la carte (Figure 2), elle représente approximativement un rectangle d'une quarantaine de kilomètres de long et d'une quinzaine de large, orienté selon l'axe nord-ouest sud-est. L'orientation générale du relief est perpendiculaire à ce rectangle: les Bornes offrent ainsi une succession de chaînes parallèles, orientées sud-ouest nord-est, d'altitudes croissantes, qui culminent à $2500 \mathrm{~m}$. À ses pieds, la vallée de l'Arly constitue une profonde entaille, tandis que, en continuant vers le sud-est et en pénétrant par là même dans le Beaufortain, on rencontre de nouveau une succession de reliefs dont les altitudes maximales s'échelonnent de $2000 \mathrm{~m}$ à pratiquement $3000 \mathrm{~m}$. On doit reconnaître enfin un caractère relativement verrouillé à l'aire en question; les débouchés de ces pays de montagne vers les grandes vallées alpines inférieures se font par des gorges ou des défilés qui rendent la circulation malaisée, tandis qu'à l'inverse, les échanges entre les vallées concernées, sont facilités par l'altitude modérée du col des Aravis et du col des Saisies. La vigueur et la qualité de ces échanges internes à la zone concernée sont attestées par le fait que, jusqu'au début du $\mathrm{xx}^{\mathrm{e}}$ siècle, le col des Saisies était un lieu réputé pour ses foires? ${ }^{7}$.

Bien que le substrat géologique de cette zone soit hétérogène, puisqu'elle se situe entre Préalpes et massifs alpins centraux, ses caractéristiques géomorphologiques de surface sont plutôt homogènes. On se situe en zone de montagne moyenne, entre $900 \mathrm{~m}$ et $1600 \mathrm{~m}$ pour l'essentiel. En Bornes, en Val d'Arly comme en Beaufortain, les vallées présentent un profil évasé qui ménage une certaine douceur à de très nombreuses pentes. La terre est argileuse et le climat connaît un fort taux de précipitations: les pâturages jouissent, à juste titre, d’une excellente réputation ${ }^{8}$. L'opposition classique, entre l'adret — au soleil comme disent les autochtones — et l'ubac — à l'envers selon l'expression consacrée localement — se traduit par celle des prairies et des versants boisés, où domine l'épicéa. Convenons toutefois que cette disposition relève de l'idéal et connaît ponctuellement des adaptations et des dérogations. Les hauteurs des montagnes, jusqu'à la limite des rochers et des falaises sommitales, sont dévolues à l'alpage. L'architecture de cette partie du paysage, aussi importante soit-elle pour l'économie locale puisque nous sommes dans une zone de transhumance pastorale, n'entre pas dans le cadre du présent article 9 . 


\section{Constances, variations et transformations : dater un peu mieux ces systèmes agricoles?}

D'un point de vue fonctionnel, le chalet savoyard répond de prime abord à plusieurs exigences: il sert d'habitat à une famille nucléaire; il fait fonction - fonction cruciale en hiver - de refuge pour les animaux domestiques, à savoir un troupeau de six à dix vaches, un cheval (qui est toujours une jument), quelques moutons et, selon la saison, une truie et sa portée (les cochons), sans oublier bien sûr les poules, les lapins, les chats (indispensables pour traquer les souris) et le chien. À cela s'ajoute la fonction, complémentaire, d'emmagasiner une masse appréciable de foin, destiné à nourrir les bêtes au fur et à mesure que l'hiver avance. Le chalet comporte enfin une cave à fromage. Notons en revanche que la conservation des grains et l'entreposage des biens précieux, tels que les vêtements féminins brodés, se faisaient à l'écart. La plupart des chalets possède en effet une annexe, appelée le plus souvent grenier et distante d'une quinzaine de mètres en moyenne. Invariablement, la raison donnée pour expliquer ce dispositif renvoie aux risques d'incendie. L'expulsion des biens les plus précieux du chalet habité garantit qu'en cas d'incendie, la famille affligée par le malheur ne perde pas tout ${ }^{10}$.

D'un point de vue architectonique, la technique de construction en bois pièce sur pièce détermine à la fois le plan, plus ou moins carré, en tout état de cause orthogonal, et les dimensions maximales de la construction, tributaires de celles des arbres utilisés. On obtient ainsi des bâtiments de gabarit assez homogène: de 12 à 15 mètres, autant en longueur qu'en largueur. La hauteur des façades s'élève jusqu'à une dizaine de mètres; la pente des toits est en moyenne de 30 degrés. Les soubassements sont toujours à deux niveaux décalés, ce qui reflète le fait qu'à quelques exceptions près, ils sont édifiés sur des terrains en pente. Le bois au contact permanent du sol étant sujet à pourrissement rapide, les chalets comportent tous une assise en pierre, dont l'importance varie toutefois sensiblement d'un type à l'autre. L'orientation plein sud, face à la pente, représente l'idéal en matière d'implantation. Il est clair cependant que tous les chalets ne sont pas construits sur l'adret, face au midi ${ }^{11}$.

C'est rendu précisément à ce point de la description que nous basculons du côté de la variation et de son potentiel de transformationnalité. Nous proposons d'en isoler les principaux axes, de discuter brièvement des implications matérielles des différences engendrées, puis de relever leur distribution spatiale. Voici les quatre traits susceptibles de variation que nous avons isolés: (1) Les dispositions respectives de l'habitation et de l'étable (appelée invariablement écurie dans les parlers locaux), étant entendu que la grange à foin se trouve toujours dans la section supérieure du chalet; (2) le dispositif d'évacuation de purin; (3) le système de circulation entre, précisément, ces différentes parties du chalet ainsi que, plus globalement, ce que nous qualifierons ici, par métaphore, l'« emballage » du chalet, soit le bardage de la structure pièce sur pièce; (4) l'indication de la date de sa construction et, éventuellement, du nom des personnes qui l'ont fait construire. Il reste en outre un dernier élément de variation à mentionner : la cheminée, qui est tantôt en pierre, tantôt en bois. Nous aurons à y revenir à l'issue d'une première analyse formelle. Pour l'instant examinons tour-à-tour les quatre moteurs de la variation. 


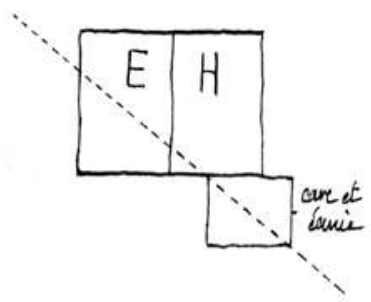

COUPE

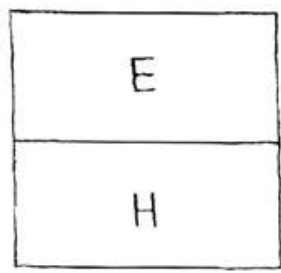

PLAN

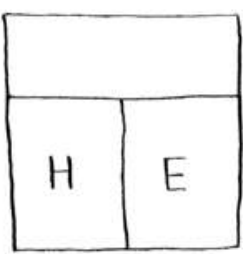

PLAN

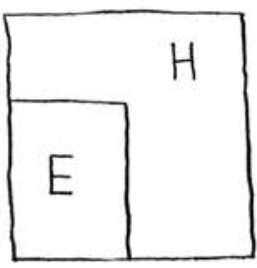

PLAN

Fig. 2.1.a

Fig. $2.1 \mathrm{~b}$

Fig. 2.2

Fig. 2.3

\section{Dispositions respectives de l'habitation et de l'étable}

Dans les Bornes septentrionales, à savoir cet arc qui se dessine de Manigot au Grand-Bornand en passant par Thônes, les Villards-sur-Thônes et St-Jean-de-Sixt, l'étable est située à l'arrière du chalet, au même niveau que l'habitation, qui elle-même donc se trouve en façade, à l'étage. En revanche, compte-tenu de la pente, l'étable est de plain-pied: elle traverse de part en part le bâtiment, jouxtant de bout en bout l'appartement. Dans certains chalets, la séparation entre l'étable et l'habitation est encore plus ténue, puisqu'elle est à claire-voie. Il s'agissait, disent explicitement les informateurs, de profiter au mieux de la chaleur que dégagent les bêtes (Figures 2.la et 2.1b).

À la Clusaz, l'étable et l'habitation se jouxtent, à l'image de ce qui se passe au nord, mais, tel au jeu de taquin, on assiste à un glissement à 90 degré des pions, puisqu'elles sont disposées parallèlement dans le sens de la longueur du bâtiment (Figure 2.2).

En Val d'Arly, nouveau glissement à $90^{\circ}$, pour l'étable seulement qui se trouve en façade, tandis que la partie habitable conserve une position largement latérale. On tend déjà toutefois vers une organisation de l'espace interne qui se rapproche de celle du Beaufortain dans la mesure où la cuisine, lieu où se trouvent l'âtre et la cheminée, est systématiquement rejetée à l'arrière de la maison (Figure 2.3).

En Beaufortain, une inversion radicale s'accomplit par rapport aux Bornes septentrionales, puisque non seulement l'étable se trouve en façade et l'habitation, à l'arrière du bâtiment, mais il y a de surcroît rupture de niveau. L'étable en effet se trouve un demi-niveau plus bas que l'habitation. Autant l'étable que l'habitation sont, ipso facto, semi-enterrées. Alors qu'au nord des Bornes, habitation et étable sont en bois, en Beaufortain, l'habitation est en pierre, comme le sont les murs latéraux de l'étable, seule sa paroi externe étant en bois, en l'occurrence en panneaux de bois et non en poutres pièce sur pièce. À cet égard, ce modèle fait également rupture avec les trois précédentes configurations, où l'étable appartient à la partie structurelle du bâtiment (Figures 2.4a et 2.4b).

\section{Le dispositif d'évacuation du purin}

Il s'agit en l'occurrence d'un chenal traversant l'étable, prolongé par un orifice carré de vingt centimètres par lequel sont poussées, grâce à un outil spécifique, le racle, vers l'extérieur des urines et des matières fécales du bétail (auxquelles anciennement étaient adjointes celles des occupants de la maison) et, enfin, d'une « fosse à purin ${ }^{12} »$ ou encore selon les endroits, sur un « tas de purin » où ce mélange est entreposé avant d'être réutilisé par la suite comme fumure. 


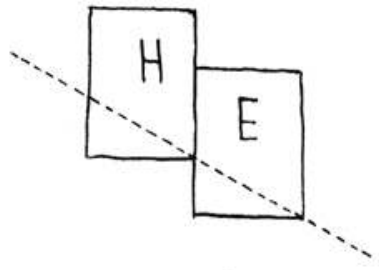

COUPE

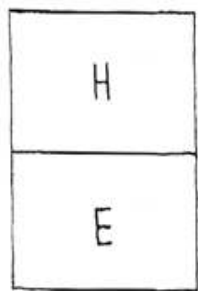

PLAN

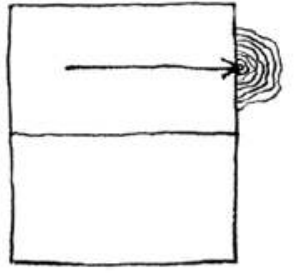

Fig. 3.1

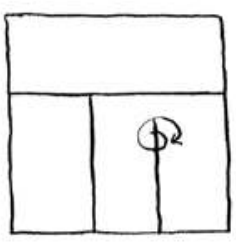

PLAN

Fig. 3.2a
Dans les Bornes septentrionales, le chenal court perpendiculairement à l'orientation principale du chalet. L'orifice d'évacuation se trouve en vis-à-vis de la porte de l'étable et la fosse, donc, à l'extérieur sur le côté opposé (Figure 3.1).

À la Clusaz, le chenal court parallèlement à l'orientation principale du chalet. Situation unique dans toute l'aire, la fosse à purin se trouve sous l'étable, c'est-à-dire sous le chalet lui-même. L'orifice d'évacuation se trouve donc dans le sol de l'étable. La fosse à purin ouvre sur l'extérieur, en façade. Elle est très souvent voutée (Figures 3.2a et 3.2b).

Dans le Val d'Arly, le chenal se brise en un T. L'orifice d'évacuation se trouve directement en façade, le purin se déversant dans une « fosse » qui peut tout aussi bien être qualifiée de « tas » et qui se trouve directement accolée à la façade du chalet (Figure 3.3).

En Beaufortain, on assiste à une sorte d'externalisation maximale du purin, puisqu'on retrouve la même disposition qu'en Val d'Arly, sauf que le chenal se prolonge à l'extérieur de la maison et coupe une terrasse, le pave, faite de grosses pierres ajustées et large d'environ un mètre cinquante. Ce qui fait que si le purin est effectivement extrait par l'avant du chalet comme en Val d'Arly, il en est néanmoins repoussé un peu plus (Figure 3.4).

En résumé, entre les Bornes septentrionales et le Beaufortain, il y a bien inversion, entre un principe d'accumulation du purin latéral contigu et un principe frontal et éloigné, la vallée de la Clusaz et le Val d'Arly jouant, là encore, parfaitement leur rôle transitionnel d'un point de vue formel. L'inversion équivaut à une rotation à $90^{\circ}$ qui passe par un point aveugle, là où la fosse latérale des Bornes vient se glisser sous le chalet à la Clusaz, opérant ainsi une parfaite transition entre l'option de latéralité et celle de frontalité qui s'incarne dans le Val d'Arly et en Beaufortain. Mais ce n'est pas tout, en Beaufortain, c'est le principe de contiguité, propre aux trois autres cas de figure, mais renforcé à la Clusaz par catagenèse, qui se voit abandonné au profit d'un principe d'éloignement, aussi relatif soit-il.

\section{Le principe des circulations et l'essentage}

Dans les Bornes septentrionales, la circulation interne aux chalets se caractérise par une série de galeries couvertes qui enserrent le bâtiment, à laquelle s'ajoute une communication directe par le biais d'une porte, entre l'étable et l'habitation, à moins qu'une simple paroi à clairevoie ne sépare ces deux espaces de vie. Plusieurs escaliers, aussi raides qu'étroits, ménagent des passages entre ces différentes galeries et/ou espaces protégés (bûcher, cave, etc.), situés à différents niveaux.

À la Clusaz, on assiste à une limitation des galeries extérieures couvertes; en revanche, on voit apparaître l'ébauche d'un système de couloirs internes, qui prend toute son ampleur en Val d'Arly. Il en résulte que les pièces d'habitation et l'étable sont distribuées par une même entrée.

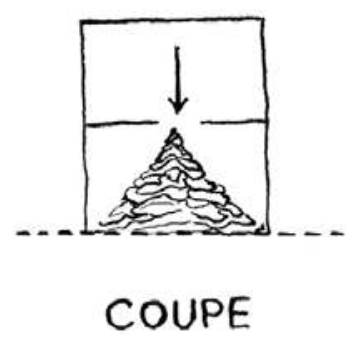

Fig. $3.2 b$

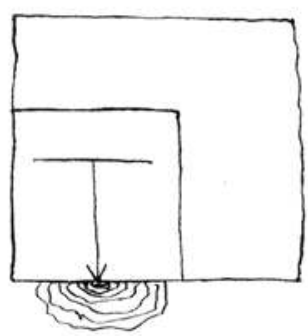

Fig. 3.3

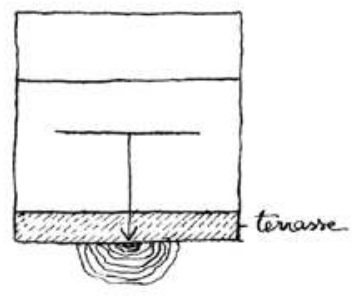

Fig. 3.4 
C'est en Val d'Arly que l'on rencontre un principe de circulation interne poussé à son maximum. Un seul couloir conduit d'une part à l'étable et d'autre part aux pièces d'habitation. Il n'y a plus de galeries frontales ou latérales. Tout au plus, voit-on parfois un minuscule balcon en façade, sur lequel nous allons revenir (Cf. page 213).

En Beaufortain, l'espace interne est totalement compartimenté. En revanche, la circulation externe est aménagée, en témoignent notamment la terrasse et le dispositif qui permet d'éloigner le tas de fumier de la façade. En réalité, tout passe par une circulation externe. Pour l'occupant de ce type de chalet, accomplir la moindre tâche ayant trait à l'exploitation impose de sortir et de s'exposer aux intempéries. En comparaison avec des chalets des Bornes qui privilégient les portes intérieures et les escaliers de toute sorte ou encore ceux de la Clusaz, ou plus encore, ceux du Val d'Arly qui abondent en couloirs et en recoins, les chalets du Beaufortain affectent une sorte d'austérité fonctionnelle et de déni de confort de leurs habitants qui tranchent singulièrement avec ce que nous avons pu observer ailleurs.

Sans vouloir céder à la tentation d'une psychologie des usages, ou plus sulfureuse encore, d'une psychologie des techniques, on serait enclin à rapprocher cette contrainte de circulation externe en Beaufortain avec l'absence de bardage, ou d'essentage pour reprendre l'expression utilisée dans les Bornes et le Val d'Arly. Précisons notre pensée. L'essentage consiste pour l'essentiel à fixer des planches par dessus la structure en poutre pièces sur pièces du chalet. Logiquement, ce sont les côtés les plus exposés aux pluies dominantes (provenant, dans toute la région, du nord-ouest) qui jouissent en priorité de cette protection supplémentaire. Cela étant, les informateurs sont ouvertement partagés sur l'intérêt du bardage, certains soulignant le fait que, si les poutres sont moins exposées à l'humidité d'origine pluviale, elles sèchent également moins bien et qu'en définitive ces planches dites protectrices favoriseraient la prolifération de vers. Somme toute, nous proposons d'y déceler plutôt un véritable principe architectonique dans la mesure où on perçoit aisément une continuité entre cet usage de planches plaquées sur la structure, parfois recouvrant la totalité du chalet, et celui qui tend à enfermer les balcons latéraux et, donc, à les transformer en galeries (Cf. page 213).

\section{La datation}

Du nord au sud de notre aire géographique, le système de datation des chalets subit enfin des variations (Figures 4, 5 et 6). Dans la petite vallée du Sapey, qui appartient à la commune de Thônes, on lit la « date de naissance » du chalet, inscrite en toutes lettres, sur le linteau de la porte de la grange, laquelle se trouve toujours à l'arrière du bâtiment. Au Grand-Bornand et à la Clusaz, cette datation demeure en revanche invisible de l'extérieur. Parfois, elle figure sur le linteau de la porte d'entrée de l'appartement, côté intérieur, ou sur le manteau de la cheminée. De façon plus caractéristique, au Grand-Bornand, elle est inscrite aussi sur la poutre verticale interne, dite "pointe d'âne », qui fait office de moise afin de maintenir le pignon supérieur de la façade avant. En Val d'Arly, la datation redevient visible: en toutes lettres, elle se déchiffre sur la panne faîtière, du côté de la façade avant. Cela étant, cette lecture doit se faire latéralement et non frontalement, comme c'est le cas dans la vallée du Sapey. L'information est en effet inscrite sur le côté de la panne. Souvent enrichie par le nom du bâtisseur et une phrase de consécration, elle déborde du reste de la panne faîtière et se donne à lire sur le côté des pannes qui la jouxtent. En Beaufortain, enfin, l'inscription orne également la panne faitière. Minimaliste, 
ne comportant que la date d'élévation du bâtiment et les initiales de ses commanditaires, parfois agrémentée d'un motif décoratif, elle se loge sur la tranche inférieure de la panne. Elle se lit donc frontalement, mais en relevant le regard à la verticale et non de biais comme c'est le cas en Val d'Arly. En résumé, on assiste de nouveau à un cycle de variations scalaires qui se résume en une inversion globale de la datation, entre une extrémité et l'autre de l'aire: date complète inscrite à l'arrière du bâtiment, sous une forme horizontale; inscription interne, invisible de l'extérieur, tantôt verticale, tantôt horizontale; datation devant le bâtiment, sous une forme déployée et à lecture longitudinale (moyen terme entre l'horizontal et le vertical), puis, enfin, sous une forme brève, à lecture verticale.

\section{Au-delà du fait technique}

Deux résultats se dégagent de notre examen de l'architecture du chalet savoyard pièce sur pièce. Premièrement, il semble vain de rattacher les variations observées à des déterminants matériels, liés à l'environnement ou à des choix techniques radicalement différents auxquels se seraient adonnés les groupes sociaux concernés. Deuxièmement, ces variations s'organisent en une polarisation aux extrémités géographiques de son aire de distribution et la présence d'éléments intermédiaires, d'un point de vue formel, entre elles. Il est à noter que les déclinaisons des formes intermédiaires ne se recouvrent pas tout à fait, engendrant en surface une plus grande diversité de combinaisons de formes - de type de chalets donc - qu'il existe effectivement de spectres de variabilité. Il nous apparaît légitime en ce sens de parler d'une grammaire des styles, expression qu'il ne faut pas prendre comme une simple métaphore, mais dont il faut accepter au contraire toutes les implications. Les variations observées et leurs ordonnancements stricts dans l'espace, qui appartiennent à la famille des transformationnalités lévi-straussiennes, prennent dans ces circonstances une valeur paradigmatique et renvoient nécessairement à une sémantique. Le chalet savoyard pièce sur pièce est-il un dispositif à signifier et, si tel est le cas, que signifie-t-il? Toutefois, avant de nous lancer à corps perdu dans une heuristique de cet ordre, nous nous sentons tenus de nous adonner à une ultime digression d'ordre technique.

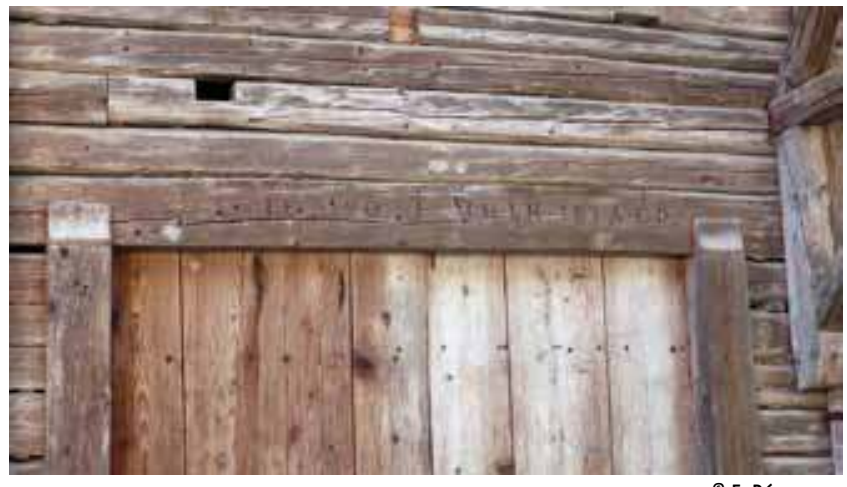

○ E. Désveaux

Thônes - Le Sapey : inscription de la date sur un linteau de porte situé en face arrière du chalet, lecture horizontale. (Fig. 4)

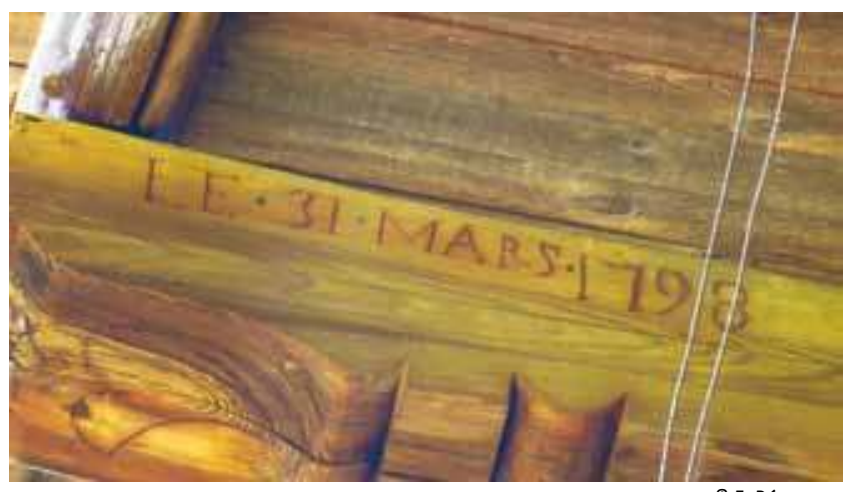

${ }^{0}$ E. Désveaux

St-Nicolas-la-Chapelle, La Poulière : inscription de la date sur la panne faîtière de la façade avant, lecture latérale. (Fig. 5)

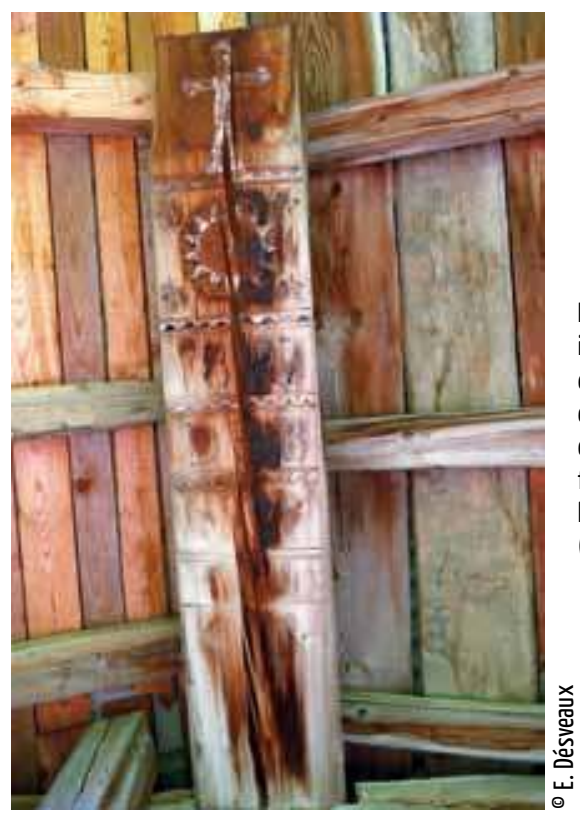

Hauteluce - Les Combes : inscription de la date d'élévation du chalet et initiales des ses commanditaires sur la panne faitière de la façade avant, lecture frontale verticale. (Fig. 6) 
La disposition respective de l'habitation et de l'étable qui s'inverse d'un bout à l'autre de l'aire est certainement le phénomène le plus remarquable de ceux que nous avons répertoriés, et, jusqu'à un certain point, on pourra admettre qu'il conditionne les autres. Or, chacune des options que nous avons évoquées sur ce plan présente des avantages et des inconvénients. Il faut savoir en effet que les vaches, leur souffle surtout, produisent une chaleur humide qui, à long terme, génère des nodules de pourrissement dans les bois. À cela s'ajoutent bien entendu les effets des éclaboussures d'urine et de matières fécales, lesquelles affectent, quant à eux, les planchers et les parties inférieures et moyennes des parois. Il est clair que le meilleur palliatif de ces attaques répétées d'humidité fétide réside dans une bonne ventilation de l'espace. Dès lors, la position de l'étable en arrière du bâtiment, telle qu'elle prévaut dans les Bornes septentrionales, semble idéale au regard de la ventilation puisqu'elle s'assortit toujours d'une situation de transversalité absolue par rapport au bâtiment. La porte de l'étable s'ouvre d'un côté et le purin est évacué de l'autre ${ }^{13}$. On observe que le trou d'évacuation du purin est surmonté, dans le fond de l'étable, d'une ou deux petites baies, qui ont vocation manifeste à alimenter un courant d'air. Cependant cette solution présente l'inconvénient de laisser s'accumuler le purin sur le côté, en un emplacement qui jouit d'un ensoleillement limité. Cela ralentit son séchage et sa transformation en fumier: il tend en conséquence à s'écouler le long du chalet, jusque sous les parties d'habitation qui sont placées devant. En bref, une certaine tenue de l'exploitation impose un travail de transfert du purin, partiellement transformé seulement (et non complètement) en un lieu de stockage intermédiaire, avant son utilisation l'été suivant. Cela représente en soi une tâche à accomplir.

La solution adaptée en Beaufortain présente une inversion des avantages et des inconvénients que nous venons d'énoncer. L'évacuation du purin, directement devant l'étable, et même, nous l'avons vu, à une certaine distance du chalet, apparaît comme idéal. Exposé en plein soleil, il sèche rapidement et son accumulation n'est jamais problématique. En revanche, l'étable semi-enterrée souffre d'un défaut intrinsèque de ventilation. Du coup, le souffle chaud des bêtes ne s'attaque pas tant aux murs de l'étable, qui sont en pierre, qu'aux poutres porteuses supérieures immédiatement adjacentes. On retrouve là le point faible de tous les chalets du Beaufortain.

À la Clusaz et en Val d'Arly, nous nous trouvons, du point de vue technique comme du point de vue formel, dans des situations intermédiaires. À la Clusaz, la fosse située en dessous de l'étable semble résoudre élégamment la question du purin. Notons simplement qu'elle impose un surcroît de travail lorsqu'il s'agit de le prélever pour le transporter ailleurs puisqu'il faut se courber pour accéder à la matière à extraire ${ }^{14}$. Surtout, tout comme en Val d'Arly, l'étable souffre d'un défaut de ventilation puisqu'elle ne jouit pas des bénéfices d'une vraie transversalité. Elle pâtit en outre d'un mauvais écoulement des fluides au sol. L'étable est en effet en étage. Du coup, le nivelage du plancher en fonction de profils ad hoc s'avère complexe à mettre en œuvre, et surtout à maintenir, à la différence de ceux d'une étable de plain-pied ${ }^{15}$. On observe ainsi dans les chalets de ces deux zones un écrasement de la façade qui correspond au fait que les poutres inférieures, celles qui sont au contact permanent avec les excréments animaux, sont très souvent en piteux état. L'exposition au soleil, latérale à la Clusaz, frontale au Val d'Arly, ne suffit pas à garantir un séchage répété et efficace de cette section de la structure. En outre, en Val d'Arly, la « fosse » à purin se trouve au contact immédiat avec la façade. Comme dans les Bornes, il faut en conséquence surveiller régulièrement son niveau afin d'éviter que purin et bois n'entrent en contact permanent et que le premier ne vienne ruiner le second. 

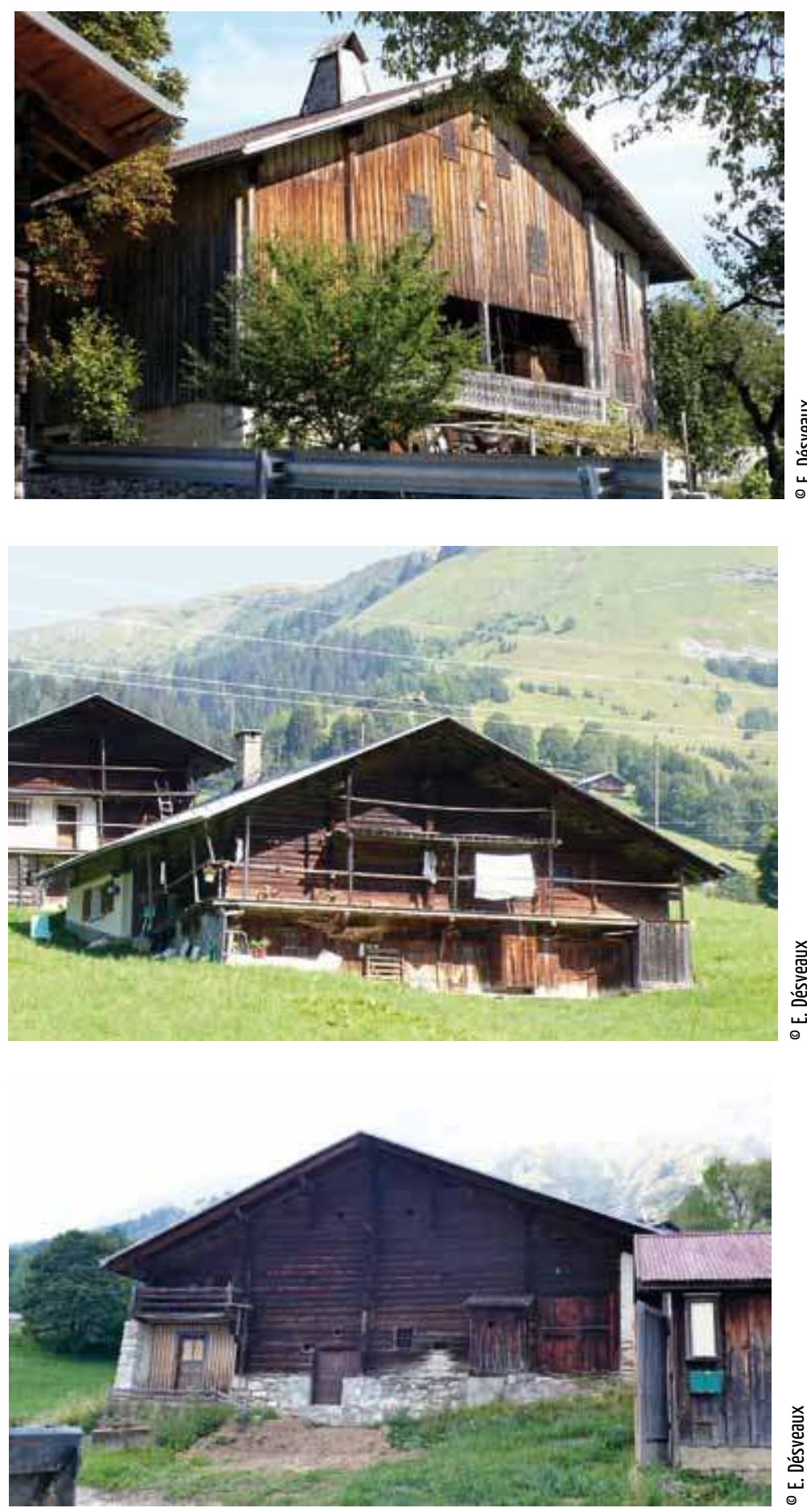

\section{Les Villards-sur-Thônes. L'essentage intégral}

L'essentage (le recouvrement des murs extérieurs par des planches), omniprésent dans les Bornes septentrionales, prend son caractère le plus accentué à Villards-sur-Thônes. Cette technique relève d'une logique de l'emballage. II confère aux chalets des Bornes un aspect apprêté, que l'on peut qualifier de chichiteux. Cette impression de chalet-bonbonnière se renforce d'autant plus que l'essentage est très couramment associé à des rambardes de balcon à balustrade ouvragée, dont le goût provient manifestement du Faucigny et du Chablais, qui s'étendent au nord-est des Bornes (Hermann 1980 : 108).

\section{Hauteluce - les Grangettes. Façades à balcons aériens caractéristiques du Beaufortain}

\begin{abstract}
En Beaufortain, les rambardes des balcons se réduisent à de simples troncs effilés que reprennent à angle droit leurs portants, traçant une quadriangulation de la façade, où en définitive l'option architectonique pièce sur pièce se donne entièrement à lire de l'extérieur, jusque - ou plutôt surtout - dans sa relation avec les parties maçonnées. On a affaire à une esthétique de la fonctionnalité affichée et du dépouillement formel qui préfigure, aux yeux de l'auteur, celle du Bauhaus.
\end{abstract}

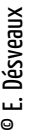

\section{La Giettaz - Les Mertines. Façade à balcon réduit}

À mi-chemin entre la Clusaz et le Beaufortain, en Val d'Arly, le système même du balcon tend à disparaître. Dans de très nombreux chalets, il n'existe pas; dans d'autres, il se rencontre à un état résiduel, dissymétrique, occupant un petit tiers de la façade.

Ces variations donnent à relativiser la rationalisation technologique. Les informateurs expliquent en effet que de grands balcons se justifient par la nécessité de faire sécher en automne les produits de la récolte des vergers et des jardins avant de les cacher pour l'hiver. Ou encore le foin, qui aurait été rentré trop précipitamment dans la grange et serait encore légèrement humide. On le ressortirait brièvement pour profiter des beaux derniers jours afin d'accomplir son séchage. Au Grand-Bornand, le balcon supérieur (car à partir d'une certaine taille tous les chalets de l'aire étudiée sont susceptibles d'avoir deux niveaux de balcon ou de galerie) est clos par des lattes horizontales disposées à claire-voie. Ce dispositif est censé répondre entièrement à cet ultime séchage du foin. Or, en Val d'Arly, ces dispositifs manquent ou sont considérablement réduits. Il y a peu de chance que les paysans de cette section intermédiaire de l'aire considérée aient eu, dans le passé, moins de besoins en termes de séchage tardifs que leurs congénères des Bornes ou du Beaufortain. En revanche, dans la logique des transformations formelles retenue ici, le phénomène d'absence est à proprement parler remarquable, puisqu'il offre une transition entre les balcons-galeries qui caractérisent les Bornes (et jusqu'à un certain point l'architecture de la Clusaz), autrement dit d'une forme entrant dans la façade, et les balcons totalement aériens du Beaufortain, autrement dit d'une forme saillante de la façade. Entre les deux, prédomine une façade lisse, sans relief, ni dans un sens, ni dans l'autre. 
Cette brève digression technique nous mène à la conclusion que, contrairement à l'idée reçue, une architecture entièrement en bois ne correspond en aucun cas à une optimalisation technique dans le cadre d'une économie principalement pastorale et ce, a fortiori, lorsque l'on opte pour le principe des poutres empilées et encastrées aux angles dans la mesure où celui-ci interdit de faire tout remplacement ponctuel de pièces abîmées par des neuves. Voilà qui renforce notre conviction de nous orienter résolument vers une interprétation sémantique du phénomène.

\section{Vers une interprétation sémantique}

Les quatre moteurs de la variation, que nous avons de bonnes raisons désormais de redéfinir comme les moments d'un groupe de transformations, nous servent, en toute logique, de guide dans la perspective d'une interprétation sémantique. Examinons-les tour à tour dans cette optique, sans perdre de vue le principe méthodologique selon lequel la signification réside dans la polarisation. La position respective de l'étable en avant ou en arrière du chalet cache la véritable opposition qui est en jeu: c'est celle de savoir si étable et habitation se situent au même niveau, ou bien sont décalées. On s'aperçoit en effet que les options des Bornes septentrionales, de la Clusaz et du Val d'Arly, s'équivalent en ce qu'elles maintiennent les hommes et les bêtes sur un même plan, tandis qu'en Beaufortain, un demi-niveau les sépare. La leçon est ici autant concrète - paroi à claire-voie entre habitation et étable, accès direct par une porte ménagée entre ces espaces ou encore partage de l'accès et des couloirs de circulation internes par les uns et les autres - que symbolique. Elle pose au fond la question cruciale que suscite le mode de vie des Savoyards de moyenne montagne, à savoir celle de la plus ou moins grande proximité dans laquelle humains et animaux vivent ou plus exactement doivent vivre. Elle pose en d'autres termes la question de l'habiter et de l'abriter. Un chalet remplit à l'évidence ces deux fonctions, mais jusqu'à quel point sont-elles séparées ou se confondent-elles? Dès lors, on comprend mieux également la différence entre les systèmes de circulation qui oppose l'option interne à l'option externe. Soit, comme dans les Bornes et le Val d'Arly, on dispose d'un accès direct aux bêtes; soit, comme en Beaufortain, on doit sortir de son chalet, le longer et finalement entrer dans l'étable, située un demi-niveau plus bas. Le système d'écoulement du purin et de son traitement entre en résonance puisque, finalement, il oppose le très rapproché à l'éloigné, toute chose égale par ailleurs. On voit dès lors fort bien se démarquer deux options fondamentales: l'intimité relative, ou le maintien à distance, certes lui aussi relatif, mais toutefois renforcé symboliquement par la hiérarchie qu’offre la différence des niveaux respectifs de l'habitation et de l'étable. En Beaufortain, les vaches sont logées plus bas que les hommes. En comparaison avec la situation qui prévaut au nord, nous dirons volontiers qu'elles sont reléguées à un niveau inférieur.

Aux yeux des paysans savoyards, les bêtes (c'est-à-dire pour l'essentiel les vaches; cela vaut également pour la jument) représentent non seulement une valeur, mais possèdent également une personnalité. Nos interlocuteurs ne cessent de le dire: « une vache, c'est comme une personne ». Leurs vaches portent un nom propre et répondent à leurs appels. Elles ont leurs habitudes, se rendant ainsi par exemple d'elles-mêmes 
à la traite. Tantôt, elles se montrent dociles, tantôt rebelles. C'est surtout autour du vêlage qu'on leur prête les comportements et des attitudes proprement humains. Elles auraient, un ou deux jours à l'avance, le pressentiment de l'imminence de la mise à bas et en éprouveraient une nervosité qu'elles seraient capables de faire partager. La durée de vie normale d'une vache s'étendait, dans le passé, sur une période d'au moins quinze d'ans, parfois même vingt ans. La relation de dépendance mutuelle et d'intimité - de solidarité et d'affection — qui se noue au cours du temps entre l'exploitant et chacune des vaches de son troupeau érode inexorablement la conscience de la différence ontologique qui le sépare d'elles. De sorte que le chalet, dans la mesure où il dramatise, dans ses différentes variations, cette problématique de la proximité et de la relégation, contribue en tant que dispositif sémantique à réaffirmer la barrière qui, sur le plan métaphysique - ou si l'on préfère cognitif — assigne les uns et les autres à une condition qui leur est propre, alors même que l'expérience quotidienne semble démentir cette séparation.

Parvenu à ce stade de notre exposé, offrons-nous une nouvelle pause technicosociologique. Nous avions évoqué brièvement la cheminée, tantôt en bois, tantôt en pierre. La première est apanage des Bornes et du Val d'Arly; sa distribution se poursuit d'ailleurs au-delà de l'aire concernée en direction du nord-est, dans le Chablais et le Faucigny. Elle heurte notre sens commun qui voit, de prime abord, une aberration dans ce choix d'un matériau inflammable pour construire la totalité d'une cheminée — manteau, hotte et conduit — d'autant que nulle part, les pierres ne manquent dans ces pays de montagne. La résolution de l'énigme par des considérations historiques qui font appel à la survivance d'anciennes traditions burgondes ne convainc guère, comme le souligne Raulin ${ }^{16}$. Écoutons plutôt ce que disent les informateurs. Selon l'un d'eux, le conduit en forme pyramidale qui vient coiffer le sta-fu, autrement dit l'âtre, présente un net avantage sur un conduit de pierre car le dispositif s'avère « autoramonant ». Le bois, même coupé, reste un matériel vivant. Il réagit aux variations de chaleur et d'humidité, ce qui provoque, à intervalles réguliers, le décollement et la chute des plaques de suie ${ }^{17}$. Paradoxalement, concluait notre informateur, la cheminée en bois est plus sûre qu'aucune autre... pour autant toutefois, reconnaissait-il, que le feu ne prenne pas trop d'ampleur. Sa puissance, maintenue en conséquence à un niveau modéré, permettait de cuisiner, de chauffer le lait pour fabriquer le fromage, de fumer des viandes, mais il n'était pas censé assurer à lui seul le chauffage de toute l'habitation. Les bêtes y contribuaient de leur côté, d'où la proximité physique qui demeurait entre étable et appartement. La philosophie d'une telle complémentarité calorique du foyer domestique et des bêtes domestiques s'exprime très nettement dans le plan des chalets du Val d'Arly - dont proviennent les commentaires sur les avantages objectifs de la cheminée en bois - où les chambres se trouvent au plus près de l'étable ${ }^{18}$, tandis que la cuisine est aménagée en arrière du chalet. En Beaufortain, la hotte de la cheminée, dont seul le manteau est en bois, possède exactement les mêmes, généreuses, proportions que celle des Bornes et du Val d'Arly car, en définitive, les fonctions primordiales de l'âtre y étaient rigoureusement identiques: cuisiner, fabriquer le fromage, fumer les viandes. Les foyers étaient-ils ici beaucoup plus vifs que ceux des Bornes? Difficile de répondre aujourd'hui à la question. Disons simplement que le caractère semi-enterré et maçonné des pièces d'habitation leur conférait une meilleure inertie que celle dont jouissaient les appartements des chalets situés plus au nord, de sorte que les choses devaient, en définitive, s'équivaloir. Par ailleurs, selon les témoignages que nous avons recueillis, il arrivait 
souvent qu'un, ou plusieurs membres d'une famille soient amenés à dormir dans l'étable. Par temps de grands froids, voire, dans certains cas, de façon plus permanente. Ce qui revient à dire que l'extrême proximité avec les bêtes n'inspirait aucune répugnance aux paysans du Beaufortain. Elle n'avait rien d'inconcevable pour eux dans la pratique. En ce sens, leurs mœurs, pour reprendre un ancien vocabulaire, ne différaient pas vraiment de celles de leurs congénères des Bornes et du Val d'Arly. On voit par là que la tension entre proximité et séparation d'avec les bêtes relève d'un registre strictement sémantique.

\section{Formule canonique des mythes et symbolisme « mou »}

Au terme de cette deuxième digression technique dont les implications dépassaient toutefois le domaine entendu de façon stricte, reprenons le fil principal de notre argument en introduisant désormais le dernier de nos facteurs de variation, à savoir la datation. Somme toute, le fait n'est ni très original, ni spécifique à notre aire d'intérêt. Dans le monde occidental, les maisons sont en effet très souvent datées. Mais là encore, c'est la variation à l'intérieur de notre aire qui devient vecteur d'intelligibilité. Or, qu'observe-t-on? Dans la vallée du Sapey, à l'extrême nord des Bornes, une « date de naissance » est livrée au jour près, comme celle d'un enfant; en Beaufortain, on se contente d'indiquer l'année d'édification du bâtiment, mais on signale en revanche l'identité des commanditaires. Ils forment, dans l'idéal du moins, un couple marié et les initiales des patronymes des deux familles sont inscrites à parité, en dépit d'une légère inflexion patriarcale (les initiales du mari précèdent celles de l'épouse). Dans les deux cas, cela revient à dire que le chalet est assimilé à un être vivant, puisqu'il naît à un moment donné. À partir de là, l'accent est mis tantôt sur sa singularité, telle celle de l'individu, tantôt sur ce que l'on pourrait appeler sa « généalogie ».

Dans l'un de ces derniers articles publiés, Lévi-Strauss propose une analyse comparative des architectures océaniennes qui débouche, en guise de clé d'interprétation globale, sur une reprise de la formule canonique des mythes qu'il avait lui-même découverte presque un demi-siècle plus tôt (Lévi-Strauss 2001). Il nous est impossible ici de discuter de façon approfondie cet outil analytique qui restait d'ailleurs en partie énigmatique aux dires mêmes de son inventeur. Nous nous contenterons d'une définition pour l'instant a minima: la formule canonique correspond à l'accomplissement d'un groupe de transformations en vertu de la double torsion sémantique qu'elle met en valeur et qui opère la transition d'un plan phénoménal à un autre ${ }^{19}$.

La formule canonique des mythes connaît plusieurs écritures possibles, dont les deux principales sont :

$$
F x(a): F y(b):: F b(x): F a-1(y) \text { ou ainsi } F x(a): F y(b):: F b(x): F a(y-1)
$$

et se lit ainsi: la fonction $\mathrm{x}$ de (a) est à la fonction $\mathrm{y}$ de (b) ce que la fonction $\mathrm{b}$ de (x) est à la fonction de $\mathrm{a}^{-1}$ (c'est-à-dire l'inverse de a) de y ou à la fonction a de $\left(y^{-1}\right)$. 
En repartant maintenant de la différence entre habiter et abriter dont nous avions établi précédemment l'importance et en retenant la notion de naissance dont la pertinence s'est rappelée à nous par le biais des datations: on peut écrire la formule suivante.

\section{Donner naissance (habiter): Produire (abriter):: Abriter (habiter): Produire (tuer)}

Formule qui se traduit en langage naturel ainsi. Donner naissance justifie le fait d'habiter, c'est-à-dire d'être, au sens ontologique, une unité familiale nucléaire, tout comme produire (du lait et, son inverse, du purin ${ }^{20}$ ) justifie d'abriter les animaux. Symétriquement, il faut habiter pour abriter (c'est toute la question de la solidarité et de la proximité entre les humains et les bêtes discutée plus haut), tout comme il faut tuer (l'inverse de « donner la naissance ») pour produire. Tout le mérite de la formule canonique est de lever le voile sur cet aspect des choses qui serait, sinon, demeuré inaperçu: afin de rester lactantes, les vaches doivent régulièrement donner naissance à des veaux et ceux-ci sont, dans leur grande majorité, tués à très brève échéance ${ }^{21}$. La mort réitérée tapisse en arrière-plan la production quotidienne du lait, volontiers perçue par ailleurs comme relevant d'une sorte d'immanence consubstantielle au chalet lui-même en tant qu'entéléchie. Il est frappant de constater combien les paysans savoyards évitent, encore aujourd'hui, à aborder cette dimension de leur activité d'éleveurs. La mort comme substitut au sevrage relève chez eux du non-dit. Il y a en effet quelque chose d'insupportable à tout mettre en œuvre pour donner naissance, pour favoriser l'émergence du vivant, et de devoir, ensuite, presque dans la foulée, procéder à son élimination ${ }^{22}$. Et nous retombons sur le thème, devenu sous cet angle douloureux, de l'éventuelle proximité ontologique entre les bêtes ${ }^{23}$ et les humains. Plus que jamais, il s'agit de conjurer les affres de l'identification.

\section{Envoi}

Tous les chalets dont il a été question ici ont été construits entre le XVII esiècle et la première moitié $\mathrm{du} \mathrm{xx}^{\mathrm{e}}$. Si les conditions historiques dans lesquelles a été abandonnée la façon de les construire nous sont bien connues - le processus s'est presque déroulé sous nos yeux - on ne sait rien de sa genèse, ni de son aire de distribution originelle. Les écrits ne s'intéressent que très tardivement à décrire ces chalets « pièce sur pièce » et les images les représentant avec suffisamment de précision ne remontent guère au-delà du $\mathrm{XIX}^{e}$ siècle. Aucun document historique ne permet de dater quoi que ce soit en la matière. Cette absence de données a "ouvert une autoroute ", si nous pouvons nous exprimer ainsi, à l'interprétation de cette architecture en terme d'archaïsme ou de survivance. Il nous semble toutefois difficile de parler en ces termes, face à une technique dont on sait, par simple relevé de la datation des bâtiments, qu'elle a donné lieu à des innovations. La principale d'entre elles réside dans la moise et le verrou qui permet une augmentation sensible de l'élévation de la façade et qui se généralise à partir de la première moitié du XIXe . 
Mais fait plus significatif encore pour notre argument, au pourtour de l'aire savoyarde du chalet « pièce sur pièce », l'architecture en bois repose sur un autre principe. L'élévation procède de poutres verticales, étayées par d'autres horizontales. Les technologues parlent alors de charpente à colonnes (Raulin 1977a : 31). Cette structure supporte les fermes de la charpente et commande le remplissage des parois par des «planches». Or, il se trouve que, dans les exemplaires les plus anciens des chalets construits selon cette dernière technique encore observables, les «planches » de remplissage se révèlent en définitive tellement épaisses qu'elles se rapprochent de la section normalisée d'un bois utilisé dans l'option pièce sur pièce. Au fond, mis à part le principe de solidarisation des bois, ce qui distingue les deux techniques ne tient peut-être pas tant à une relation d'antériorité, donc supposément d'infériorité technique, de l'une par rapport à l'autre, mais à ce que l'une utilise des troncs d'arbre dans pratiquement toute leur longueur, tandis que l'autre, au contraire, exige de les couper afin d'obtenir des planches destinées à venir se loger entre les montants pour faire panneau. Dans le parler local, on désigne les chalets construits selon cette technique, en armature ou en vitraux (autrement dit, en ce qui concerne la dernière expression, dont l'aspect évoque celui des vitres et de leur cadre). Celle-ci permet incontestablement de construire des bâtiments plus grands, telles ceux que l'on découvre en remontant la section supérieure du Val d'Arly, à Praz-sur-Arly, à Megève et, en redescendant vers Sallanches, à Combloux. Cela étant, la différence entre les deux options techniques ne renverrait-elle qu'à la taille des bâtiments, qui, elle-même, renverrait alors à des données de nature sociologique? Sans exclure une explication de ce type, dont les développements dépasseraient en tout état de cause le cadre du présent article, nous suggérons volontiers que nous avons presque « sous la main » une autre explication, d'ordre sémantique: le chalet pièce sur pièce reflète le souci d'enserrer le bon équilibre ontologique entre proximité et éloignement d'avec les bêtes, entre production de vie et nécessité de mort, dans un espace non pas tant confiné que circonscrit grâce à des éléments vitalistes ayant préservé leur intégrité: en l'occurrence des arbres demeurés pratiquement intacts du bas jusqu'en haut. Car la pensée dispose ainsi des moyens de résoudre l'équation entre le près (la souche) et l'éloigné (la cime de l'arbre), le vivant (l'arbre dans son intégralité) et la mort ponctuelle (l'arbre a été coupé; il a été abattu, il est tombé). Les informateurs insistent toujours sur le fait que le bois est un matériau qui n’a jamais été rendu totalement inerte, qui reste en partie vivant: «le bois, ça craque, ça bouge toujours ».

Le bois, lequel? Il s’agit en réalité de l'épicéa, espèce qui se dit localement la pesse. Les choses se dénouent dès lors en termes de division des rôles et des sexes. Si l'homme et la femme s'occupent à égalité des bêtes au jour le jour, les tâches typiquement domestiques, en particulier entretenir le feu et faire la cuisine, celles qui relèvent spécifiquement de l'habiter, sont, sans surprise, féminines. La construction et l'entretien du chalet, celles qui relèvent donc de l'abriter, reviennent en propre aux hommes. Il est temps de rappeler alors que le mot chalet, vient de cala, et signifierait, selon l'école étymologique allemande, un « endroit abrité » (geschützte Stelle) (Wartburg 1922 - 2002 : [2] II - 1 : 50) et de revenir à une ultime considération technique. Les toitures des chalets étaient recouvertes d'ancelles, sortes de longues tuiles en bois. Celles-ci, au contact de la neige et de la pluie, sont soumises à un processus de pourrissement assez rapide. Elles devaient être retournées ${ }^{24}$, puis renouvelées régulièrement. Traditionnellement, la principale activité des hommes à la veillée d'hiver résidait donc dans la fabrication d'ancelles neuves. Ils fendaient à l'aide d'un départoire (une sorte de hache à longue lame), outil appelé localement le ferancelle ${ }^{25}$ 
— mot masculin par excellence - des billes de pesse - mot féminin en parler local — prédécoupées à l'avance. Somme toute, il était de la responsabilité des hommes d'entretenir le chalet et cet entretien, en tant qu'activité réitérée, avait en définitive trait essentiellement au toit. Nous n'avons jamais entendu parler d'une femme qui serait montée sur un toit pour en changer les ancelles ou, par simple déplacement, en améliorer l'étanchéité. C'est à proprement parler inconcevable.

L'habiter et l'abriter sont placés respectivement sous l'égide du féminin et du masculin. Nous sommes dans une relation en bouteille de Klein (Lévi-Strauss 1985). Car si l'abriter englobe manifestement l'habiter, l'habiter reste in fine la raison d'être de l'abriter. Il y a par ail-

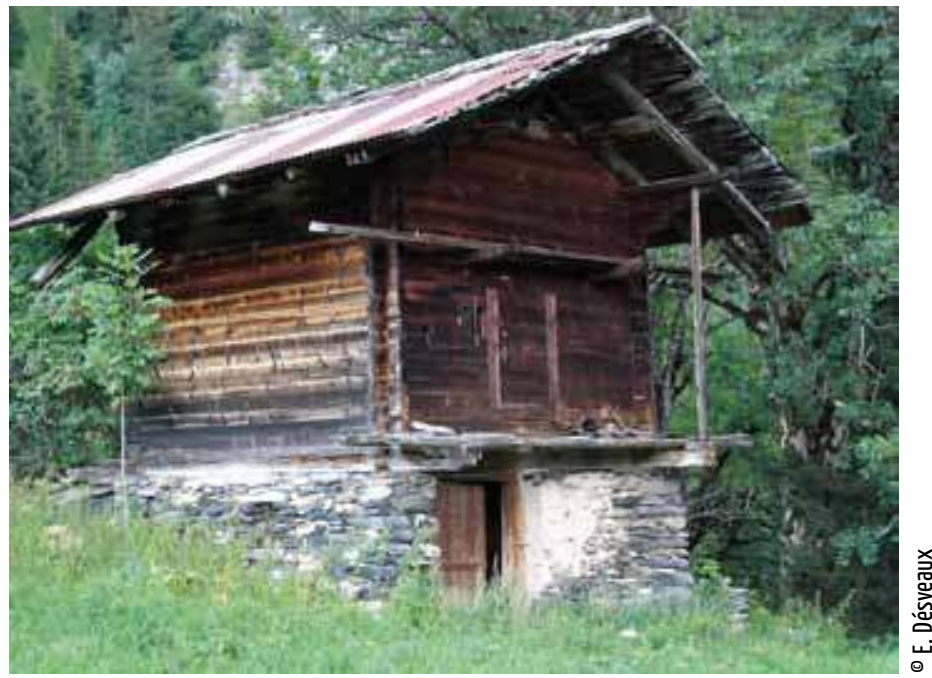

Hauteluce - Les Lanches Un grenier à l'abandon.

(Fig. 7)

et à la femme: la richesse à l'état brut. On comprend mieux désormais la nécessité du grenier comme entité séparée dans l'espace physique (Figure 7). Son contenu est à la fois neutre et inerte. Il n'a pas sa place dans le chalet, espace bâti à la fois hypervitalisé et hypersexualisé. Une ancienne croyance disait qu'à la suite d'un accouchement, la délivrance devait être enterrée dans la cave « parce qu'il fallait que ce soit dans la maison » (Canova 1998). Or, qu'est-ce que le placenta sinon un synonyme de vie, son doublon presque, et ce, en lien direct avec la sexualité?

\section{$\&$}

Cet essai sur l'architecture des chalets poutre sur poutre de la Savoie septentrionale cherche à dépasser une perspective strictement formelle ou esthétique comme celle d'Abraham (1964), ainsi que celle technico-écologique de Raulin, avatar hybride de la géographie humaine à la française et de l'écologisme culturel américain (Arbos 1922 ; Netting 1981). Aspirant à une interprétation sémantique, elle se place sous le signe de Lévi-Strauss et de sa formule canonique des mythes. Les lecteurs qui nous auront suivi jusqu'ici y auront détecté quelques réminiscences du célèbre texte que Bourdieu avait consacré à la maison kabyle (Bourdieu 1970). Mais en définitive, elle doit surtout à Haudricourt qui professait que la forme prise par la relation entre les humains et leurs animaux domestiques offrait l'une des meilleures clés d'interprétation de celles qui prévalaient entre eux (Haudricourt 1962 ${ }^{26}$. Pour autant qu'ils aient des animaux domestiques, évidemment. Car ailleurs, c'est nécessairement une autre affaire. 


\section{NOTES}

Cette recherche a profité en septembre 2010 d'un financement de l'ANR, par le biais du programme PerSemSoc (Perception Sémiotique et Socialité du Sens).

Photo d'ouverture : Le Grand-Bornand, Le Planay. Chalet maintenu en l'état - La fixation des ancelles sur le toit se fait par des fins troncs élagués, à noter également la cheminée en bois (dite localement Bourne, de la racine allemande Bruna, «le conduit» qui a donné en allemand moderne Brunnen : la fontaine).

1. Lacception du mot chalet ne se limite pas aux chalets d'alpage comme le veut une tradition bien implantée chez les géographes. C'est un terme d'usage courant qui désigne en Savoie toute construction individuelle en bois. On l'oppose aujourd'hui à la villa, maison en brique ou en moellons de facture récente.

2. Voir l'étonnant avant-propos du Tableau de la géographie de la France, Paris, 1911. Citons : «L'homme a été, chez nous, le disciple longtemps fidèle du sol ».

3. H. Raulin, op. cit. et du même auteur, L'architecture rurale française, Corpus des genres, des types et des variantes: Dauphiné, Paris, 1977. Ces deux ouvrages comprennent une préface signée de Jean Cuisenier et ayant valeur programmatique en vue de définir une algèbre des types et des variantes. Rappelons que le projet qui livrera par la suite encore une vingtaine ouvrages, mené sous l'égide du musée national des Arts et Traditions Populaires, reprenait sur des bases intellectuelles totalement nouvelles, une enquête sur les habitats ruraux mise sur pied par Georges-Henri Rivière pendant la seconde guerre mondiale dans le cadre des « chantiers intellectuels de Vichy ». Pour une discussion du contexte, nécessairement ambigu compte tenu de l'époque, de ce premier projet, voir Régis Meyran, «Écrits, pratiques et faits. L'ethnologie sous le régime de Vichy », L'Homme, 1999, tome 39 $n^{\circ} 150: 203-212$.

4. Comment ne pas y voir l'écho du reste de l'onde de choc provoquée par le livre-manifeste de Bernard Rudofsky, Architecture Without Architects, publié pour la première fois à New York en 1964, tout comme au travail, exactement contemporain du précédent, et lui aussi insistant avant tout sur une perception visuelle des phénomènes, signé Raimund Abraham et Josef Dapra sous le titre Elementare Architektur (Salzburg, non daté, mais publié aussi en1964). Notons, qu'à la différence de Rudofsky qui a une ambition comparatiste universaliste, Abraham et Dapra se focalisent uniquement sur les Alpes.

5. Notre méthode doit beaucoup aux facilités offertes par les progrès accomplis récemment en matière de photographie digitale et accessoirement, par le développement du réseau routier. Ce dernier rend aisément, et donc rapidement, accessible un grand nombre de sites (rassurons le lecteur hygiéniste: nous avons aussi pas mal marché !). Ces conditions sont nouvelles; elles diffèrent de celles qu'avait connues Raulin et qui l'avaient conduit à l'exercice monographique, autrement dit à choisir, en vue d'en fournir la description la plus précise possible, un nombre restreint de bâtiments comme étant chacun représentatif d'un type. Sans vouloir trop critiquer le travail de Raulin, dont nous sommes largement tributaire et auquel nous vouons une réelle admiration, il est apparu qu'en définitive sa méthode comportait une bonne dose d'arbitraire. Comment décider que tel ou tel bâtiment pouvait être représentatif d'un type qui lui-même restait à définir? Il apparaît en outre que l'enquête de Raulin comporte de grosses lacunes dont on peut penser qu'elles sont dues autant à des contraintes factuelles qu'à des aveuglements théoriques. Concernant l'aire qui nous importe ici au premier chef, elle ne livre par exemple aucune information ni sur la Clusaz, ni sur le Val d'Arly.

6. Soit les communes des Clefs, de Manigod, de Thônes, des Villards-sur-Thônes, de St-Jean de Sixt, du GrandBornand, de la Clusaz (dans les Bornes), de la Giettaz, de Flumet, de Chaussice, de St-Nicolas-la-Chapelle, de Notre-Dame-de-Bellecombe (en Val d'Arly), d'Hauteluce, de Beaufort et de Villard-sur-Doron (en Beaufortain).

7. La foire était éventuellement le lieu de saisies par les fiscaux, les représentants de l'État (de Savoie, sarde ou français).

8. Les conditions climatiques qui prévalent dans les Alpes du Nord françaises se traduisent aux altitudes concernées par un enneigement durable durant l'hiver. Les premières neiges qui « tiennent » apparaissent en novembre et la fonte débute à la mi-mars et dure jusqu'en mai, selon les altitudes et l'exposition des versants, selon les années également. La présence de la neige sur le sol réduit considérablement les déplacements humains et immobilise de facto le bétail.

9. Il s'agit en effet d'une région où prévaut le phénomène typique des Alpes Centrales: regroupements au début de l'été des troupeaux familiaux en vastes troupeaux et montée en alpages (dit parfois estives). Ce modèle de transhumance restreinte se double, en Beaufortain notamment, d'un système de remues qui conduit les familles à occuper différents chalets 
à différentes périodes de l'année, selon un étagement saisonnier. Notons enfin que l'économie traditionnelle était centrée sur l'élevage, même si elle donnait lieu, parallèlement, à une petite polyculture d'autosubsistance reposant essentiellement sur le seigle et la pomme de terre.

10. Le grenier se trouvant parfois dans une telle proximité de la bâtiment principal, à trois quatre mètres de lui, voire étant totalement intégré à lui, qu’un trouble certain sourd dans l'esprit de l'observateur extérieur quant aux vertus salvatrices de l'éloignement, dès lors qu'il peut se révéler, dans certains cas, si dérisoire.

11. Lorsque l'exposition s'avère moins favorable, notamment en cas de construction sur l'ubac, il y a recherche du meilleur compromis entre les deux impératifs de respecter l'axe de la pente et de jouir d'un ensoleillement maximal, optant tantôt pour celui du matin, tantôt pour celui du soir. Là où les pentes sont les plus douces, comme à Notre-Dame-de-Bellecombe dont l'essentiel du territoire se situe à l'ubac, on assiste à une oscillation des implantations, certains chalets s'orientant vers le soleil, d'autres respectant d'abord l'impératif de la pente.

12. En Beaufortain, le purin ou le fumier se dit la druzde. On serait tenter de penser que le genre gammatical exprime une polarisation, d'ordre sexuel, des productions du chalet, la druzde, mot féminin, répondant au lait, qui en patois se dit lacea tout en restant un mot masculin.

13. En général, à l'est dans la vallée de Manigot, à l'ouest ailleurs.

14. Le caractère vouté des fosses à purin de la Clusaz intrigue par sa singularité. Cette forme semble n'avoir aucun équivalent. Pourtant, on peut y voir la transformation des pressoirs que l'on rencontre dans le vallée voisine de Manigot. Ici, le pressoir est une pièce semi-enterrée en façade, voutée et close, qui se trouve en général à gauche (lorsqu'on fait face au chalet). À la Clusaz, la fosse, espace semi-enterré, vouté et ouvert, se trouve exactement au même niveau, mais occupe une place symétrique, à droite du bâtiment. On remarquera que le pressoir est le lieu on l'on transforme du solide en liquide (des pommes en cidre), la fosse celui où du liquide se transforme en solide (le purin en fumier).

15. Les étables comportent toujours des planchers; il est clair que ceux qui reposent à même le sol sont plus faciles à ajuster ou à renouveler que ceux qui sont solidaires de la structure du chalet.

16. H. Raulin, L'architecture rurale française, Corpus des genres, des types et des variantes: Savoie, op. cit. p. 39. pour une discussion de ce type de cheminées.

17. Si un conduit en bois semble un défi à la raison pratique, précisons, pour atténuer cette impression, qu'il est confectionné en planches chanfreinées, technique qui le rend parfaitement étanche. La réalisation de ces cheminées est donc sophistiquée, ce qui laisse encore plus perplexe face à l'explication en termes de survivance d'un archaïsme (Cuchet 2005).

18. Le terme dialectal pour étable étant böu, il est frappant de noter que la chambre à coucher y était désignée couramment par l'expression böu a le zan, autrement dit «étable aux gens».

19. La formule canonique des mythes apparaît pour la première fois sous forme imprimée en 1955 (cf. Anthropologie structurale, Paris, Plon, 1958 : 227). Selon J.-P. Luchelli, elle aurait été conçue dès 1952 (Lacan et la formule canonique des mythes, Les temps modernes, $n^{\circ}$ 660, sept-oct. 2010). La revue L'Homme lui a consacré un dossier entier sous la direction de Jean Pouillon et la nôtre (n 106-07, 1988). Concernant notre interprétation et nos usages de la formule, voir E. Désveaux Quadratura americana, op. cit.: 36-61.

20. Inversion du clair et du sombre, du comestible et l'incomestible; cela étant, lait et purin sont analogues en ce sens qu'ils ont tout deux vocation de passer dans un délai assez court de l'état liquide à l'état solide (fromage, fumier).

21. Aujourd'hui les veaux sont, rapidement après la naissance, vendus à des engraisseurs venus de l'extérieur. Cette tractation se fait à la sauvette, comme si elle était honteuse. Notons qu'une vache donne entre dix et quinze veaux dans son existence. Or, dans l'optique de la stabilité de l'effectif du troupeau, un seul survivra.

22. Sur la culpabilité face à la mort animale, voir la très belle étude de N. Vialles (1987).

23. On remarquera que la jument jouit d'un traitement un peu différent de celui des vaches. Dans toute la région concernée, les gens ne prenaient pas en charge eux-mêmes la (re)production des équidés (chevaux, ânes, mulets). Il y avait des éleveurs spécialisés pour s'occuper de cela. Dans les Bornes, l'écurie se situe à côté de la cave, un niveau en dessous celui de l'étable. Il y a donc dissociation nette entre la jument et les vaches. En Val d'Arly, elle se trouve en vis-à-vis de l'écurie, les deux espaces étant séparés par un couloir (mais les vaches et la jument n'empruntent pas la même entrée, ce sont plutôt les humains et les vaches qui le font). En Beaufortain, l'écurie se trouve à côté de l'étable. Comme cette dernière, elle ouvre directement sur l'extérieur, en façade. Dans le cadre de notre analyse, on dira que la jument incarne en tout état de cause la polarisation maximale dans le spectre proximité/séparation. Cela est d'autant plus remarquable que les informateurs insistent volontiers sur la très grande complicité qui pouvait se nouer, à travers le travail quotidien, entre une jument et son propriétaire, la durée de vie « active » de la première pouvant aller au-delà de vingt-cinq ans. 
24. Retourner l'ancelle permet de l'« user » par des deux extrémités. Les ancelles sont superposées sur les deux tiers de leur surface. C'est, bien sûr, le tiers exposé aux éléments qui se dégrade. Une ancelle type mesure 70 $\mathrm{cm}$ de long; la largeur varie de 20 à $30 \mathrm{~cm}$, l'épaisseur entre 1,5 et $2,5 \mathrm{~cm}$. Il faut environ 16 ancelles pour couvrir un mètre carré, soit 3600 pour une toiture de 200 mètres carrés, surface moyenne d'un toit de chalet. Une ancelle dure en moyenne trente ans (15 ans environ de chaque côté). Lancelle usagée est brûlée dans l'âtre domestique.

25. Comprendre le «fer-à-ancelle».

26. Voir aussi, dans une perspective similaire, l'exemple normand étudié par F. Zonabend (1994).

\section{RÉFÉRENCES}

Abraham, Raimund J. \& Dapra J. 1964 Elementar Architektur. Salzburg: Residenz Verlag. (non daté)

Arbos, P. 1922 La Vie pastorale dans les Alpes françaises. Paris: A. Colin.

Bourdieu, P. 1970. La Maison kabyle ou le monde renversé, 739-758. In P. Maranda, Échanges et communications: mélanges offerts à Claude Lévi-Strauss. Paris: Mouton, 739-758.

- 1979 La Distinction, Critique sociale du jugement. Paris: Minuit.

Bucaille, R. \& al. 1987 Pigeons de Limagne: approche éthologique et interprétation ethnologique. Clermont-Ferrand: Université Populaire de Clermont-Ferrand.

Canova, O. 1998 Les Carnets d'une accoucheuse en Beaufortain. Montmélian: La Fontaine de Siloë.

Cuchet, X. 2005 Les Sens de l'évolution technique. Paris: Édition Léo Scheer.

Désveaux, E. 2001 Quadratura Americana, Essai d’anthropologie lévi-straussienne. Genève: Georg.

Haudricourt, A.-G. 1962 Domestication des animaux, culture des plantes et traitement d'autrui, L'Homme, 2 (1) : 40-50.

Hermann, M.-T. 1980 Architecture et vie traditionnelle en Savoie. Paris: Berger-Levrault.

Lévi-Strauss, C. 1958 Anthropologie structurale. Paris: Plon.

- 1985 La Potière jalouse. Paris: Plon.

- 2001 Figures en sablier. In P. Maranda (dir.) The Double Twist. From Ethnography to Morphodynamics. Toronto: University of Toronto Press, 15-32

Luchelli, J.-P. 2010 Lacan et la formule canonique des mythes, Les Temps modernes, $n^{\circ} 660$.

Meyran, R. 1999 Écrits, pratiques et faits. L'ethnologie sous le régime de Vichy, L'Homme 39 (150) : $203-212$.

Netting, R. 1981 Balancing on an Alp, ecological change and continuity in a Swiss mountain community. Cambridge: Cambridge University Press.

Raulin, H. 1977a L'Architecture rurale française, Corpus des genres, des types et des variantes: Savoie. Paris: BergerLevrault.

- 1977b L'Architecture rurale française, Corpus des genres, des types et des variantes: Dauphiné. Paris: BergerLevrault.

Rudolsky, B. 1964 Architecture without Architects. New York: Museum of Modern Art.

Tomasi, E. 1977 Historische Gehöftformen : In Oesterreichischer Volkskundeatlas, 6. Lieferung / 1. Teil, bl. 96, 97, $98: 1-136$

Vialles, N. 1987 Le Sang et la chair: les abattoirs des pays de l'Adour. Paris: Éditions de la Maison des sciences de l'homme.

Vidal de la Blache, P. 1911 Tableau de la géographie de la France. Paris: Hachette.

Wartburg, W. von 1922-2002 Französisches Etymologisches Wörterbuch.

Weiss, R. 1959 Häuser und Landschaften der Schweiz. Zürich: Eugen Rentsch Verlag.

Zonabend, F. 1994 Gestion de la parenté, gestion du troupeau. In F. Héritier \& E. Coppet-Rougier (Dir.) Les complexités de l'alliance, IV, Économie, politique et fondements symboliques. Paris: Archives contemporaines, $29-46$. 


\section{RÉSUMÉ}

De Lévi-Strauss à Haudricourt. Promenade architecturale en Savoie. Cet essai sur l'architecture savoyarde en bois de type pièce sur pièce repose sur l'observation d'une série d'inversions formelles qui ne répondent ni à une déterminiation écologique, ni à une déternination strictement sociologique. Une interprétation en termes sémantiques est proposée. Ce type d'habitat, dans sa variabilité, est le reflet d'une interrogation ontologique portant sur la proximité adéquate entre humains et bétail. L’analyse conduit dans un premier temps à établir la différence sémantico-fonctionnelle entre l'abriter et l'habiter et, dans un second temps, à comprendre la séparation entre espace hypervitalisé et hypersexué d'une part — la maison principale —, espace de la neutralisation sexuelle et de la perpétuation (non spéculative) de la richesse d'autre part — le grenier.

\section{ABSTRACT}

From Lévi-Strauss to Haudricourt. Architectural walking in Savoy. This essay on the vernacluar wood architecture of Savoy is based on the observation that a specific kind of building — log on log construction - offers a series of formal inversions which seem disconnected from any kind of ecological, or strictly sociological determination. Instead, we propose an interpretation in semantical terms. With its variations, this type of housing raises the ontological question of the appropriate distance between humans and cattle. The analysis presented here aims at a) establishing a semantical-functionnal differentiation between shelter and habitation and b) underlining the separation between the hypervitalized, because hypersexuated, space of the main house and the space of sexual neutralisation and of (unspeculative) perpetuation of wealth, represented by the grainery.

\section{MOTS CLÉS}

Architecture vernaculaire, ontologie, économie pastorale de montagne, Savoie

\section{KEYWORDS}

Vernacular architecture, ontology, pastoral economy, Savoy 